\title{
Proposing a Scheme for Human Interactive Proof Test using Plasma Effect
}

\author{
Akbas Ezaldeen Ali * \\ Nidaa Flaih Hassan
}

Received 8/7/2018, Accepted 16/1/2019, Published 2/6/2019

This work is licensed under a Creative Commons Attribution 4.0 International License.

\begin{abstract}
:
Human Interactive Proofs (HIPs) are automatic inverse Turing tests, which are intended to differentiate between people and malicious computer programs. The mission of making good HIP system is a challenging issue, since the resultant HIP must be secure against attacks and in the same time it must be practical for humans. Text-based HIPs is one of the most popular HIPs types. It exploits the capability of humans to recite text images more than Optical Character Recognition (OCR), but the current text-based HIPs are not well-matched with rapid development of computer vision techniques, since they are either vey simply passed or very hard to resolve, thus this motivate that continuous efforts are required to improve the development of HIPs base text. In this paper, a new proposed scheme is designed for animated text-based HIP; this scheme exploits the gap between the usual perception of human and the ability of computer to mimic this perception and to achieve more secured and more human usable HIP. This scheme could prevent attacks since it's hard for the machine to distinguish characters with animation environment displayed by digital video, but it's certainly still easy and practical to be used by humans because humans are attuned to perceiving motion easily. The proposed scheme has been tested by many Optical Character Recognition applications, and it overtakes all these tests successfully and it achieves a high usability rate of $95 \%$.
\end{abstract}

Key words: Human Interactive Proofs, Optical Character Recognition, Plasma effect, Web security,

\section{Introduction:}

Human Interaction Proofs (HIP) or also known as Completely Automated Public Turing test to tell Computers and Humans Apart (CAPTCHA), is a standard method to decide whether an employer is human or machine. It is used for web-sites to protect it from behaviors of malicious, such as, auto post, account enumeration spamming, etc. HIP requests employers to complete a simple job, which is made-up to be easier for humans but harder for machines (1).

HIP s come in many formulae, mostly it's based on text is used that basically an image presentation that encompasses alphanumeric characters positioned visible and degradation or distortion spread over background of these images, and this cannot be recognized by Optical Character Recognition (OCR). Thus, a decent excellence CAPTCHA is strong and safe. A diversity of HIP has been suggested and applied, such as labeling of image, recognition of text, recognition of audio, recognition of video, solving puzzle, identification of object (2).

Department of Computer Science, University of

Technology, Baghdad, Iraq.

*Corresponding author: $110034 @$ uotechnology.edu.iq
HIPs or CAPTCHAs based on text are practically used in actual applications, characters are intentionally distorted in text, and they are linked to prevent bots recognition. Enhance the security of text CAPTCHA could be done by methodically embedding distortion and noise, and positioning characters more firmly. These actions could make the characters harder for machine to recognize (3).

In this paper, a new suggested HIP animated video is created based on building a new plasma environment, thus it can be used practically easy by human, but it's hard to be attack by a machine.

\section{Literature Review}

The issues of Human Interactive Proofs (HIPs) have grabbed the attention of several researchers; the following section presents a brief description to some of these researches which used CAPTCH based on text with different applications and scopes:

In 2004 (4) approaches are developed to create information implanting visually textured (static and dynamic). Static patterns are designed by subtle changes on edges of original text image, while dynamic patterns are designed by using spatial-temporal constructions encompassing image 
edges. Also, handwriting is used for HIP testing, in (5) Two handwritten word recognizers are proposed, they are accomplished by taking the advantage of the difference between humans and machine in recognizing handwritten word images. In (6) an algorithm is proposed to distinguish between Persian and Arabic human user by using the features for each Arabic and Persian script, in this algorithm, random Arabic and Persian meaningless words are created and kept as image format, meanwhile, by applying some alterations, is presented to the user to ask him/her to categorise the displayed word. Another handwritten CAPTCHA images technique is presented by (7) to improve the security of web, this technique produces colored handwritten letters and words image, where randomness is associated within image to increase complexity of recognition. By exploiting the limits of Arabic OCRs in reading Arabic script in (8), Arabic CAPTCHA scheme is generated to be useful in Arabic countries, also to be beneficial in protective resource of the internet. The text-based CAPTCHA model in (2) is generated by a mixture of mathematical algebraic equations and alphanumeric words. Scenarios are created differing in Font types, alteration of alignment, and alteration of Location. CAPTCHA based simple text method is proposed in (9). This method is forbid replay of earlier submitted test (certainly not be reused), thus for whenever the page is made or refreshed, the attitude features is altered. An instance of features are: CAPTCHA's code is a series of uppercase and lowercase characters and numbers, these characters are created by randomizing compound functions, also string code are alternated by different angles and code length is diverse. Dissimilar from machinelearning-based methods that require a huge manually-labeled volume of genuine CAPTCHA to perform an in effect solver, generic and effective text CAPTCHA is presented in (10). This method requires considerably less genuine CAPTCHA but produces a greatly improved presentation.

From the above, numbers of limitation are determined such as some of the works need computational resources to be allocated, since significant amount of storage is needed to carry out these techniques, also recognizers are limited by specific languages or handwritten writing, in addition, some of these techniques do not include handwritten sentences and phrases.

\section{Human Interactive Proofs (HIPs)}

A Human Interactive Proof (HIP) is a proof that a human can create with no special tools, but which a machine cannot easily create. Commonly, HIPs are a wide type of test/answer protocols which allow an unassisted human to verify as an adult or a human in one of a certain group. Such proofs must stand against passive attacks: a party that realizes that the proof should not be capable to falsely become a proof of membership (11).

HIPs are progressively used to guard facilities against automatic script bouts. Examples of such facilities are: E-mail (spam), online registrations, ticket/event reservations, online voting, login, chat rooms, and weblogs. Yahoo, Microsoft, Ticket Master, Register.com, and Google presently are using HIPs to guard their online facilities. To be active, the HIP must be hard enough to discourage script attacks (12). This is done by raising the calculation and/or progress charges of breaking the HIP to an unprofitable level. Simultaneously, the HIP must be easy enough not to detect humans from using the facility (13).

The statute of HIP should be solved simply by user but not by a bot. HIP specifications are as follows:

1. A machine judge should be as an alternative of human.

2. All humans recognize the HIP and pass the test, whereas no computer program can pass it (14).

HIP aids to stop bots from accomplishing malicious actions like spamming and other fake actions. These bots are considered as a core threat to web services. A large number of free accounts are registered automatically by Web-bots and then use these accounts to spam valid users by:

- Sending scrap E-mail

- Decelerating the service by repetitively signing many accounts at the same time

- Causing other denial of services. Hence most of the websites have adopted HIP as defensive scheme against such Web-bots.

The current text-based HIPs are not safe as computer-vision techniques progress so fast. The improved solutions are still in the beginning since they are either hard to solve and expensive to accomplish or easy to be intruded due to compromised security. This indicates that continuous efforts are required to improve the robustness of HIP (15).

\section{Types of HIP}

HIP is considered as a reply test to the user, they are categorized on the basis of what is partial, i. e. whether characters, digits, or images. Most of popular types of HIP are:

1. HIPs created from text.

2. HIPs created from an image.

3. HIPs created from audio.

4. HIPs created from a video.

5. HIPs created from puzzle (16). 
1) HIPs created from a text: HIP based on text is considered to be very easy to be implemented. It is accomplished by representing case insensitive letters in a fixed and distorted environment. The HIP that is created from a text is very well-known in websites, as examples: Yahoo, Gmail, YouTube, and Hotmail. Figure 1 shows an example of the HIP that is created from English letters (17).

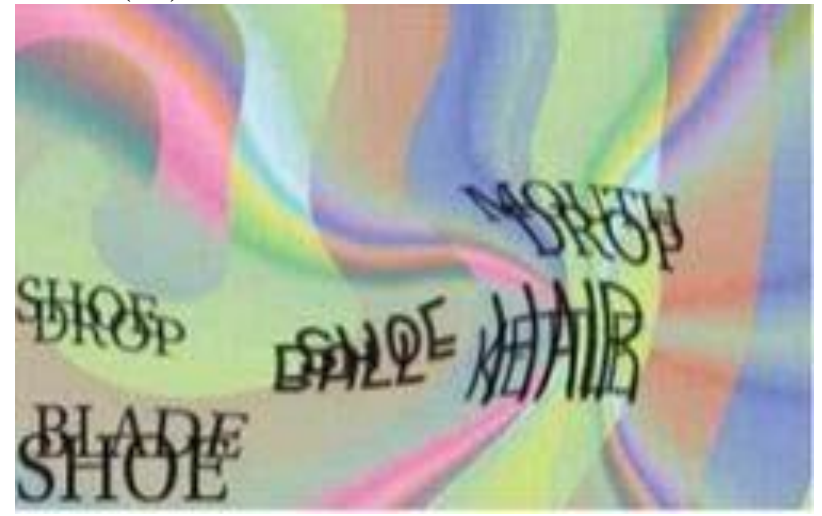

Figure 1. HIP created from text (17)

2) HIP created from image: HIPs that are created from graphics is considered to be a challenging test since the users have to detect similar images. HIPs that are created from images require image identification that is done by the user. HIP created from an image is difficult to be broken by pattern recognition technique since the pattern recognition is hard task (Artificial Intelligence) AI problem. Figure 2 shows an example of HIP created from the image (18).

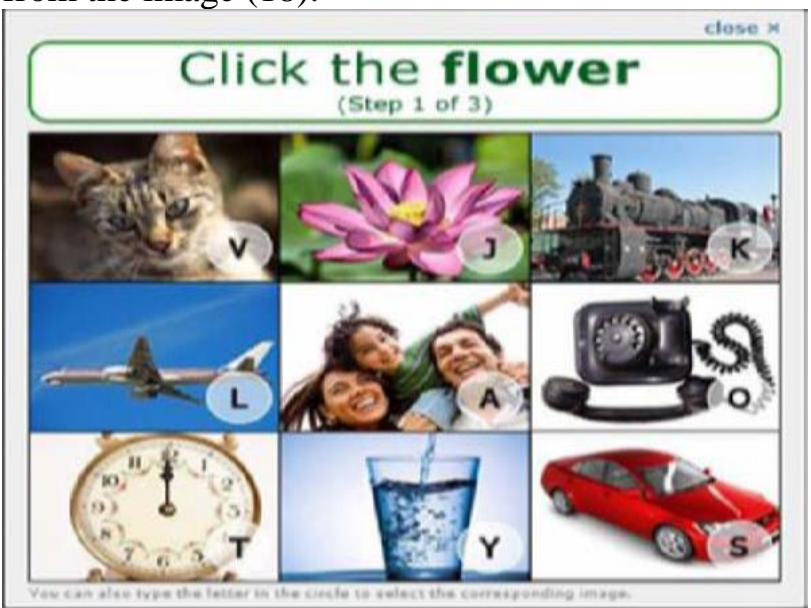

Figure 2. HIP Based on Images (16)

3) HIPs created from audio: HIPs which are based on audio are working on the sound based systems. User who cannot see clearly is able to use audio- based HIPs. An audio clasp is downloaded so that the user should listen to these clasps and recognizes the word to be submitted. ECO was the first HIP audio system put into operation at City University in Hong Kong (17).
4) HIPs created from a video: These HIPs use videos instead of texts or images or audios. In HIPs which are based on a video, users watch (You Tube) videos, the video asks the users to write descriptive keywords tags. Users watch and then properly tag keywords. This type of HIP is classified on a correct matching of user tag with truth database tags of a video. Figure 3 shows an example of HIP based on a video.

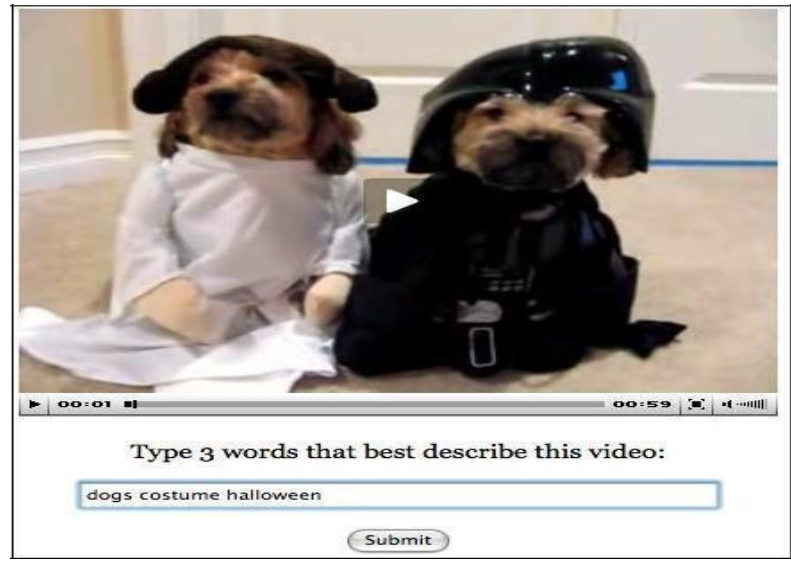

Figure 3. HIP Based on Video (19)

5) HIPs created from puzzle: HIPs which are based on a puzzle is done by presenting a picture divided into chunks, so the user collects these chunks depending on imagination to produce complete picture similar to the original picture. Figure 4 shows an example of the HIP which are created from puzzle (16) (23). J

\section{Put the puzzle together.}

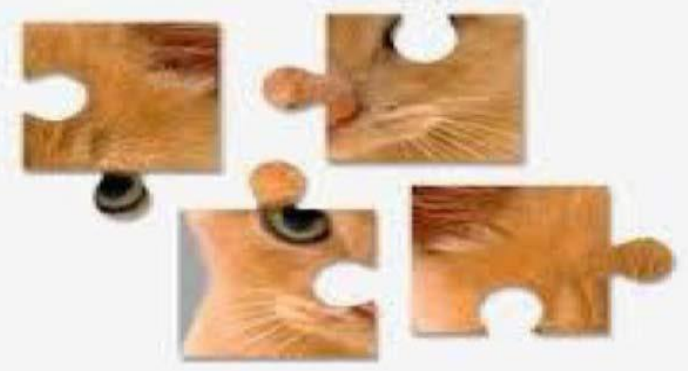

Figure 4. HIP Based on Puzzle (16).

\section{Building the Plasma}

The plasma effect is a wobbly animated visual effect which can be implemented in software rendering by using Sine function and pseudo color palettes.

There are many ways to build plasma like effects. In the past days they were created with a recalculated color table. These days, the same effect can almost be produced in real time with Sine and Cosine with cycles of a changing color warped in numerous ways to grant an illusion of organic and liquid movement (20). 
So the Plasma effect, used in this paper, is a function of Sine's, which evolves over time, in every frame. This function will take a $2 \mathrm{D}$ point as a parameter, and returns a real number. This result must be calculated for each pixel with applied color.

The Plasma function has a period of 2- pi, (where $\mathrm{pi}=3.14159265359)$, which means that it will be repeated every 2-pi until infinity. The argument of Sine function can be restrained to $0-2$ pi. Also, the Sine function returns a value in the interval $[1,-1]$, so it grow over this interval; the following sections describe this process: (20), (21)

\section{Calculation of Sine's Function}

The continuity of Sine's function calculation depends on how the plasma evolves over time. Different parameters of $\mathrm{x}$ and $\mathrm{y}$ could be used to change the Sine function in each frame, this makes Sine calculation easier, also these parameters could be selected from frame pixels. The results of this calculation are stored in a buffer to be corresponding to a specific frame at each time, as illustrated in Algorithm 1:

\begin{tabular}{|c|c|}
\hline $\begin{array}{l}\text { Algorithm } 1 \text { : The plasma } \\
\text { calculation } \\
\text { Input : } x, y \\
\text { Output : plasma function }\end{array}$ & function \\
\hline $\begin{array}{l}\text { Step_1: Index }=0 \\
\text { Step_2: Time }=\text { Get Time }() / 50 \\
\text { is used to compute plasma_function } \\
\text { Step_2: For } \mathrm{i}=0 \text { to max-y } \\
\text { For } \mathrm{j}=0 \text { to } \max -\mathrm{x} \\
\text { video_buffer( index } \\
\text { plasma_function }(\mathrm{x}+\mathrm{j}, \mathrm{y}+\mathrm{i}) \\
\text { Increment Index by one } \\
\text { End For } \mathrm{j} \\
\text { End For } \mathrm{i}\end{array}$ & // Time \\
\hline
\end{tabular}

A more attractive and wobbly plasma can be generated by combining two or more precalculated plasma functions (22), as shown in Fig.5.

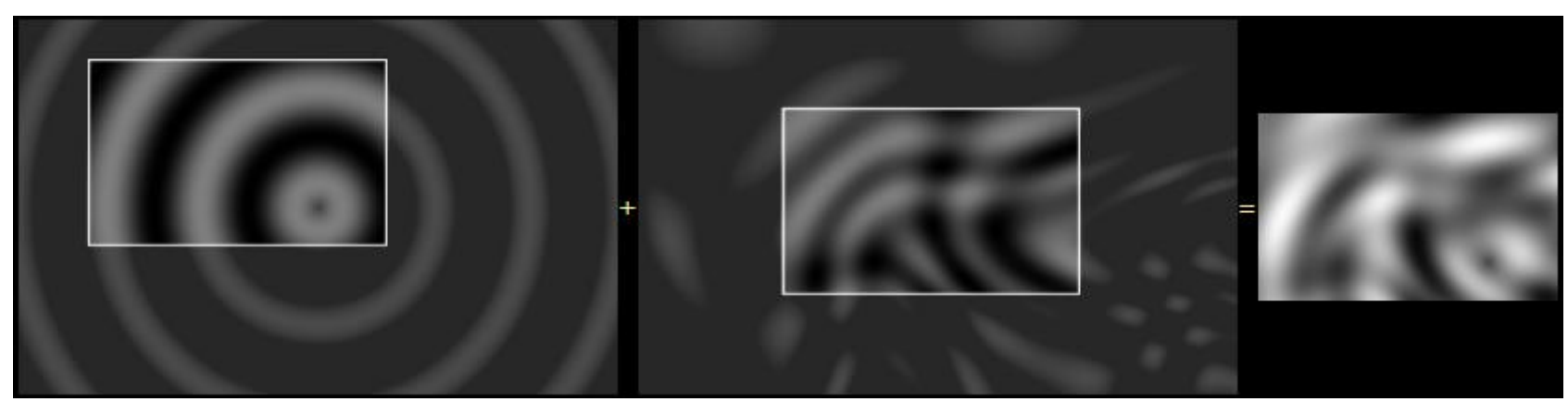

Figure 5. A Plasma effect results from combining two functions (22)

There are no limits to the functions that can generate the plasma (plasma_function), for example, define our plasma function as a combination of the three functions: F1, F2, and F3. The first Function (F1) is commonly used in calculating plasma function by applying Sine to the distance between the two points (x+ time, y) and $(\mathbf{1 2 8 , 1 2 8 )}$, where it is calculated as follows:

\section{F1= Sine(dist(x+Time, $y, 128,128) / 8)$} ..... 1

Where $\operatorname{dist}(\mathrm{x} 1, \mathrm{y} 1, \mathrm{x} 2, \mathrm{y} 2)$ is the Euclidean distance between $(\mathrm{x} 1, \mathrm{y} 1)$ and $(\mathrm{x} 2, \mathrm{y} 2)$ points.

While the second and third functions (F2 \& F3) can be calculated by applying Sine to the distance between another two points, as illustrated in the Eq. 2 and Eq.3:

$$
\begin{aligned}
& \text { F2 }=\text { Sine }(\operatorname{dist}(x, y+\operatorname{Time} / 7,192,64) / 7) \ldots . .2 \\
& \text { F3 }=\text { Sine }(\operatorname{dist}(x, y, 191,100) / 8 \quad \ldots .3
\end{aligned}
$$

The new function can be calculated by combining the three functions (F1, F2, F3) and taking their average. This is getting pretty close to the required effect $(20,22)$.

\section{Assigning Colors}

To get cool looking for the produced plasma, the color must be added to the Sine functions. The assigned color should not appear in discontinuities in order to preserve the organic and fluid look of the produced plasma.

Keeping in mind that there are 256 color palette, before displaying the plasma, an overflow test must be accomplished on the resulted color to make sure it's in the palette's range

An easy way can be used to determine what colors to assign to each result of the plasma function. This method can be achieved by taking the obtained Sinus value, and to use it to generate the RGB components of the color. Many color palettes can be generated. Some examples of these palettes are listed below: 
$\left.\begin{array}{l}\operatorname{RED}=\operatorname{Sine}(\mathrm{i} / 32 * \pi) \\ \operatorname{GREEN}=\operatorname{Sine}(\mathrm{i} / 64 * \pi) \\ \operatorname{BLUE}=\operatorname{Sine}(\mathrm{i} / 128 * \pi)\end{array}\right] \ldots \ldots 4$

Where the values of RED, GREEN and BLUE, are rounded and normalized to an integer value between 0 and 255 , and the value of $\mathrm{i}$ is iterated from $0-255$ for each palette.

Eq. 4 will produce the following palette:

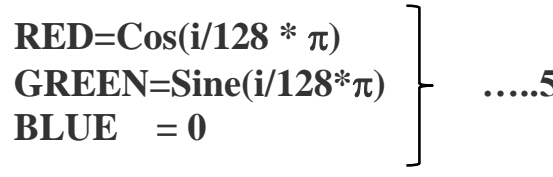

Eq. 5 will produce the following palette:

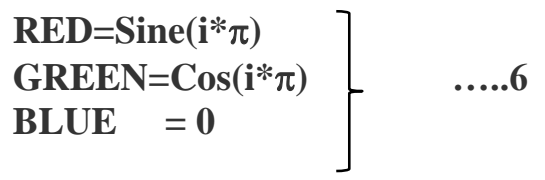

Eq. 6 will produce the following palette:

RED $=$ GREEN $=$ BLUE $=\operatorname{Sine}\left(I^{*} 5^{*} \pi\right) \quad \ldots \ldots 7$

And , Eq. 7 will produce the following palette:

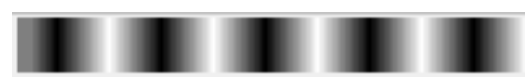

By combining the functions of the animation and the palette, the result will be different plasma effect that differs in colors and shapes. Figure 6 shows the combination of plasma effect with the color palette.

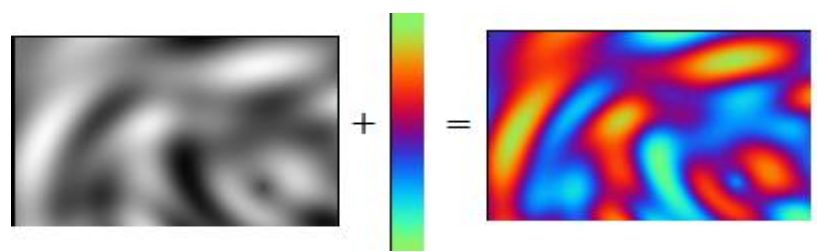

Figure 6. Plasma effect results from combining Sine function with color palette.

Since there are no limits to the imagination, many plasma effects can be generated by inventing any number of functions, recalculating them, and combining them together.

\section{The Proposed HIP Validation Test}

The basic idea, with the HIP, is to come up with a test that current technology level spam bots cannot pass, and only humans can pass it. So there is a need to make it difficult for computers to identify the code in the text, and to increase the strength of security to confuse Optical Character Recognition (OCR) programs.

In this proposed scheme, moving text in a dynamic environment is used to achieve robust secure HIP. This can be done by joining interpreted information, in particular, the changing of the words color, and the dynamic motion of Plasma background and the HIP text. The procedure that is needed to generate this scheme is as follows:

- The first step of the proposed Scheme is achieved by generating a random HIP validation text that consists of a combination of alphanumerical and numerical characters. Algorithm 2 illustrates the process of generating the proposed HIP test.

\begin{tabular}{|c|}
\hline \\
\hline $\begin{array}{ll}\text { Input : } & \text { HIP text } \\
& \text { Plasma effect that is generated by } \\
\text { applying } & \text { Algorithm } 1 \\
& \text { n: Length(HIP text ) } \\
\text { Output : } & \text { HIP validation test }\end{array}$ \\
\hline $\begin{array}{l}\text { Step_1: Select HIP validation test text, and store it } \\
\text { as a session text for the } \\
\text { subsequent HIP validation test. } \\
\text { Step_2: For each frame of the plasma effect video } \\
\text { do the following steps: } \\
\text { Call algorithm (1) to generate the current } \\
\text { plasma effect for current frame } \\
\text { For } \mathrm{i}=1 \text { to } \mathrm{n} \\
\text { next location in plasma frame } \\
\mathrm{x}(\mathrm{i})=\mathrm{x}(\mathrm{i}-1)+\text { random }(0-5) \\
\text { if } \mathrm{x}(\mathrm{i})>\mathrm{w} \text { then } \mathrm{x}(\mathrm{i})=\mathrm{x}(\mathrm{i})-\mathrm{w} \\
\text { the width of the screen } \\
\quad \mathrm{y}(\mathrm{i})=\mathrm{y}(\mathrm{i}-1)+\mathrm{w}) \mathrm{w} \text { is } \\
\text { if } \mathrm{y}(\mathrm{i})>\mathrm{h} \text { then } \mathrm{y}(\mathrm{i})=\mathrm{y}(\mathrm{i})-\mathrm{h}) \\
\text { the height of the screen } \\
\text { choose the color for next character of HIP } \\
\text { by applying one of the } \\
\text { Eq. } 4, \text { Eq. } 5 \text {, Eq. } 6 \text { and Eq. } 7 \\
\text { Insert HIP(i) into the current plasma frame } \\
\text { at location (x(i), y(i)) with current color } \\
\text { End For } \mathrm{i}\end{array}$ \\
\hline
\end{tabular}

The following are two examples of the propose Scheme HIP test by applying Algorithm 2:

\section{Example_1:}

By choosing palette that is produced by Eq. , the snapshots of the produced HIP test video are shown in Fig. 7. 


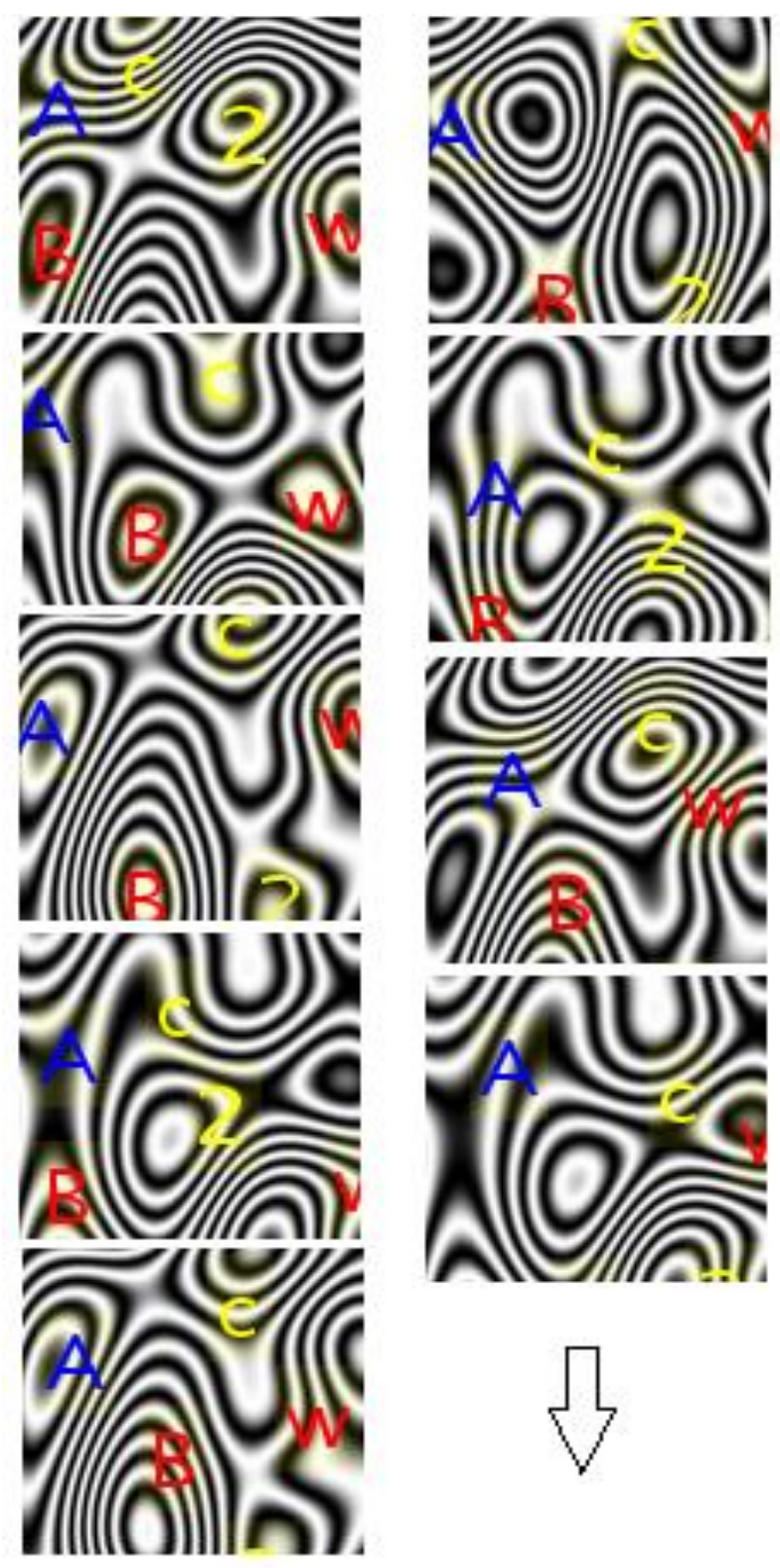

Figure 7. Some screenshots of the HIP of example1

\section{Example_2:}

Choosing palette that is produced by Equation 6, the snapshots of the produced HIP test video are shown in Fig. 8.

The animation of the HIP text with the motion of the plasma can achieve good security features, because of the difficulty of recognition by software, specifically Optical Character Recognition. In contrast, the animation makes the test far easier for humans to be solved, because humans are attuned to perceiving motion.

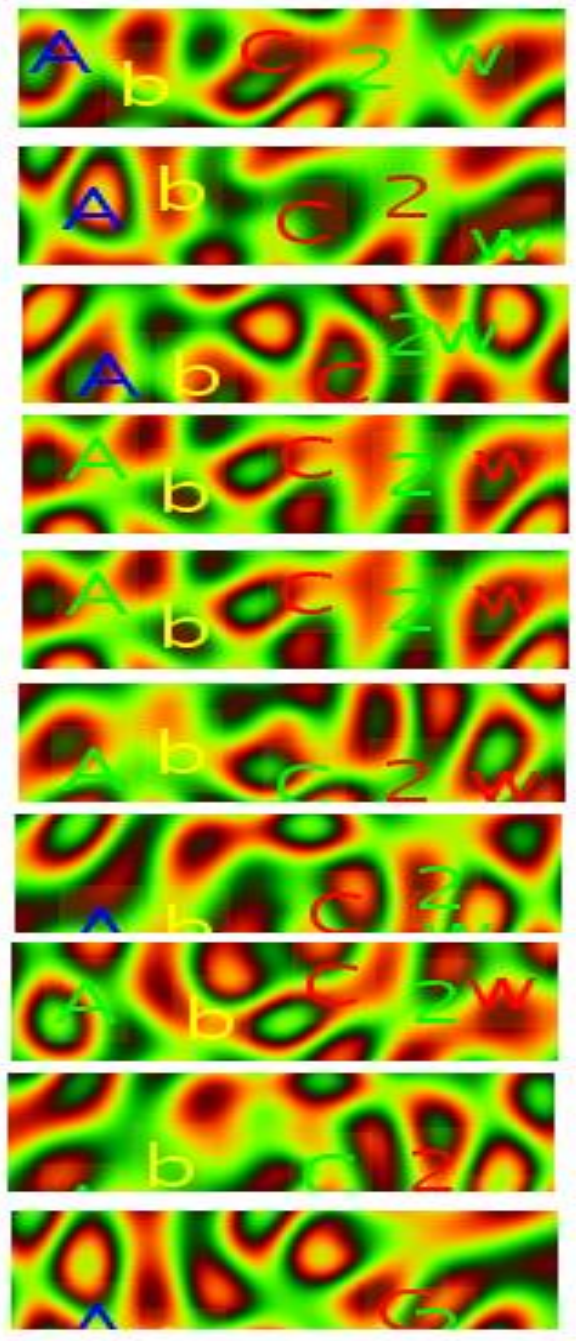

Figure 8. Some screenshots for the HIP of example2

\section{Results and Discussion:}

To make sure that our proposed scheme is secure, robust, and can resist any attack aims to break its underlying protocols via man-in-the middle, many experiments have been carried out. They are:

1) Test by using some edge detection algorithms: This test is done by taking a screenshot, converting the produced image into binary, and then applying some Optical Character Recognition (OCR) algorithms. The OCR is the process of detecting, segmenting and identifying characters from image (24). In computer vision, segmentation refers to the process of partitioning a digital image into multiple segments. Many techniques are used to segment an image; one of these techniques is the edge detection algorithm. Sobel and Prewitt edge detection algorithms are applied on the produced image to detect edges and distinguishing the text from overall screenshot as follows: 
Sobel edge detection algorithm test: By applying Sobel algorithm. The result will be as in Fig. 9 .

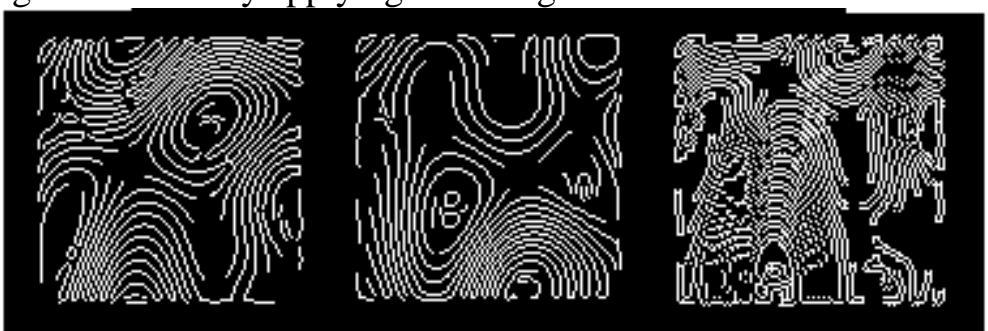

Figure 9. Applying Sobel edge detection on three frames of the HIP of Example 1

Prewitt edge detection algorithm: By applying Prewitt algorithm, the produced result will be shown as in Fig. 10.
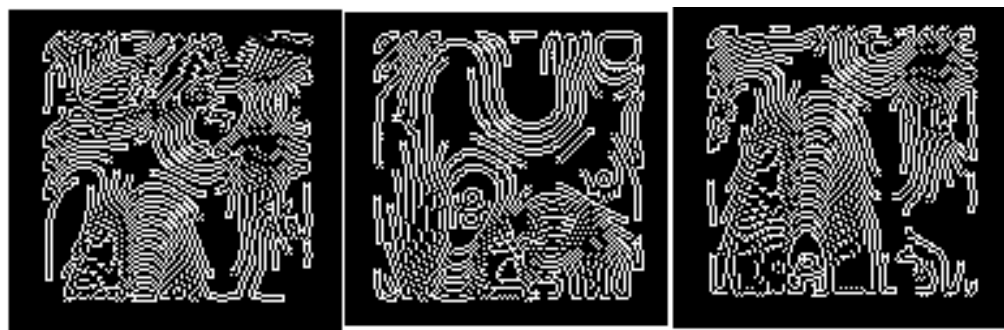

Figure 10. Applying Prewitt edge detection on three frames of the HIP of Example 1.

From Fig. 9 and 10, it's observed that the overlapping of the plasma with the characters of HIP generates chaos and confusion that makes it difficult to recognize the characters as it is clear in the following tests:

2) Tests by using some OCR applications: Several known OCR applications have been used to recognize the characters on the proposed HIP, these algorithms are implemented by using :

a. Matlab code.

b. Online OCR software.

c. An offline application which is called: Free OCR.

d. Mobile phone application which is called Text Scanner OCR.

The results of the above mentioned tests fail to recognize the characters in the proposed HIP image. Fig. 11, 12, 13 show the output of the OCR test.

The proposed scheme override all the tests of edge detection and OCR applications successfully with $100 \%$, in such that all applied programs failed to detect and recognize the HIP's characters.

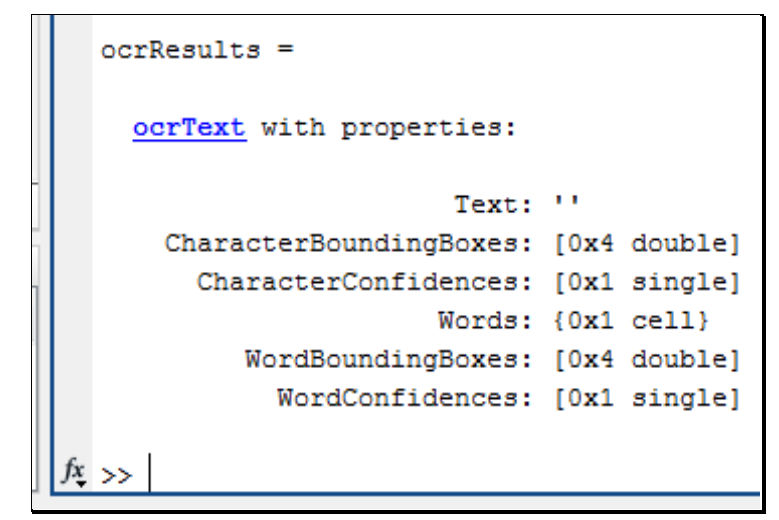

Figure 11. The output is: - null characters recognized (using Matlab code).

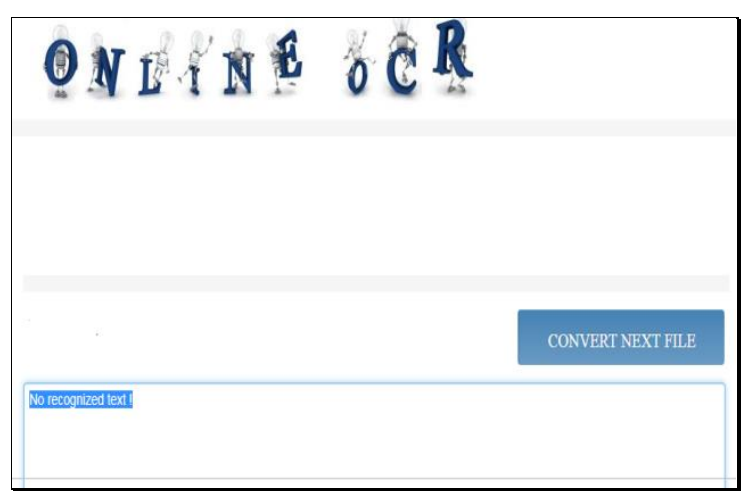

Figure 12. The output is: - No recognized text, (using Online OCR software) 


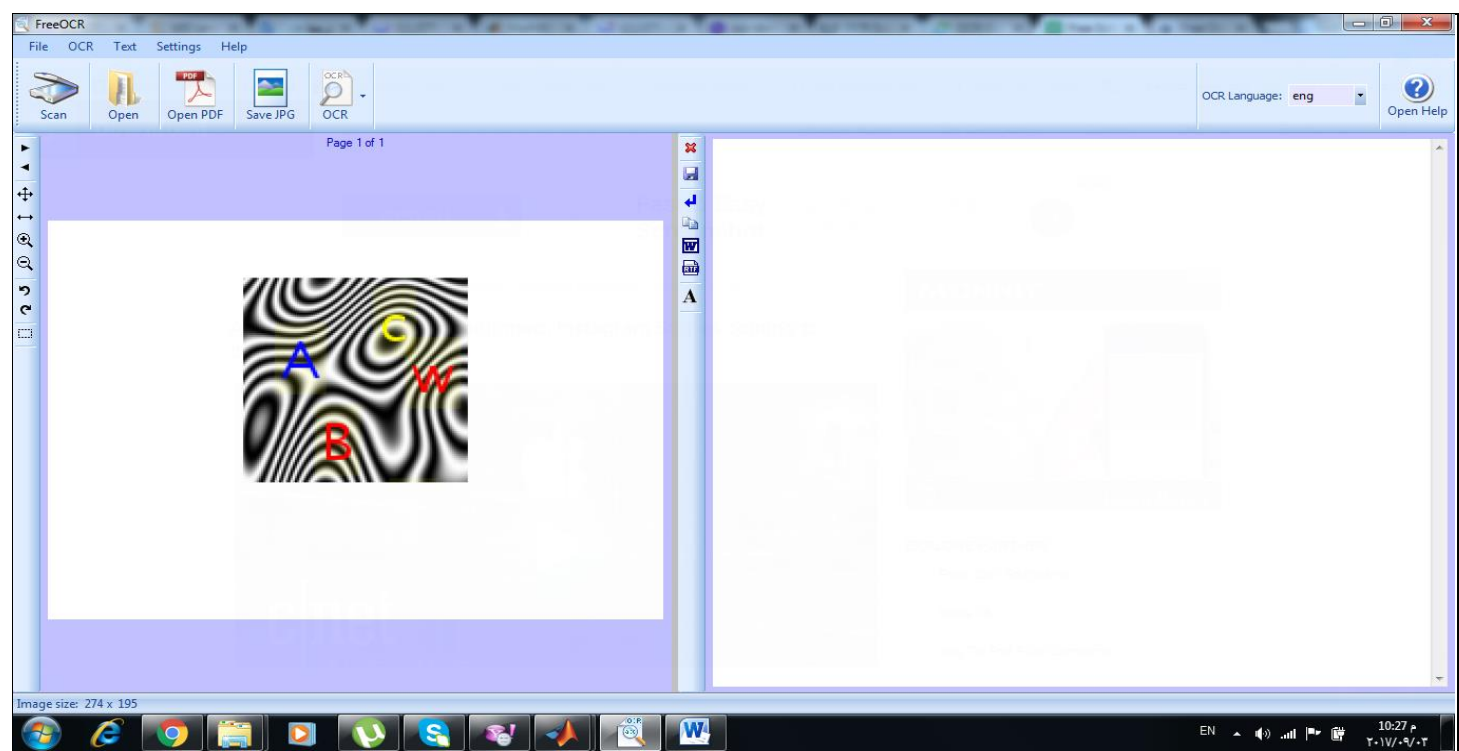

Figure 13. The output is: - no characters recognized, (using Offline Free OCR)

An important term for evaluating the quality of the proposal scheme is the usability, where several HIP tests are tested by many people.
Table 1 shows the usability results of the proposed scheme which are tested by several human users (every human user has 8 tests). The results were positive since the success rate is $95 \%$.

Table 1. Samples of people who participate HIP test

\begin{tabular}{llllllllllll}
\hline $\begin{array}{l}\text { User } \\
\text { Number }\end{array}$ & Sex & Test_1 & Test_2 & Test_3 & Test_4 & Test_5 & Test_6 & Test_7 & Test_8 & Test_2 \\
\hline User_1 & Male & pass & pass & Pass & pass & pass & pass & pass & pass & pass \\
User_2 & Male & pass & pass & Pass & pass & fail & fail & pass & pass & pass \\
User_3 & Male & pass & pass & pass & pass & pass & fail & pass & pass & pass \\
User_4 & Female & pass & pass & Pass & pass & pass & pass & pass & pass & pass \\
User_5 & Male & pass & pass & Pass & pass & pass & pass & pass & pass & pass \\
User_6 & Female & pass & pass & Pass & pass & pass & pass & pass & pass & pass \\
User_7 & Female & pass & pass & Pass & pass & pass & pass & pass & pass & pass \\
User_8 & Female & pass & pass & Pass & pass & pass & pass & pass & pass & pass \\
User_9 & Female & pass & pass & Pass & pass & pass & pass & pass & pass & pass \\
User_10 & Male & pass & pass & pass & pass & pass & pass & fail & pass & pass \\
\hline
\end{tabular}

The proposed HIP scheme is highly usable since it is easy for humans to provide the response successfully, in addition, it is not difficult for humans to visually perceive these characters despite the distortions and the motion of HIP's characters

\section{Conclusion:}

In this paper a new scheme is proposed that concentrates on designing an HIP test which is effectively resistant to attacks of spam bots programs. This scheme exploits the gap between the usual perception of human and the ability of computer to mimic this perception and achieve a more secure and more human usable HIP. The scheme could prevent attacks since it's hard for the machine to distinguish characters with animation environment displayed by digital video, but it's certainly still easy and practical to be used by humans. The strength of the proposed method compared with other methods comes from the use of the wobbly visual animated effect of plasma with the moving text's characters, which will be able to naturally resist any attack methods of static OCR recognition. The proposed scheme overrides all tests successfully by $100 \%$. These tests imply two types the first one is the edge detection tests (by Sobel and Prewitt), and the second one is done by OCR applications tests (by Matlab code, Online OCR software, Free OCR and Text Scanner OCR mobile phone application). The proposed scheme achieves a high usability rate of $95 \%$.

\section{Conflicts of Interest: None.}

\section{References:}

1. Von A, Manuel B, Nicholas JH, John L. CAPTCHA: Using hard AI problems for security. In Advances in Cryptology-EUROCRYPT [cited 13 May 2003]; (2656): 294-311. Springer Berlin Heidelberg. 
2. Kiranjot K, Sunny B. Designing a Secure Text-Based CAPTCHA. 3rd International Conference on Recent Trends in Computing (ICRTC-2015) [Internet]. 2015; (57): $122-125$.

DOI: $10.1016 /$ j.procs.2015.07.381

3. Simran S, Nidhi S. Survey of Text CAPTCHA Techniques and Attacks. International Journal of Engineering Trends and Technology (IJETT) -2015 April; 22(6):.240-245.

4. Wen-Hung L, Chi-Chih C. Embedding Information within Dynamic Visual Patterns. International Conference on Multimedia and Expo (ICME), IEEE [Internet]. [Cited 2004 June 27-30]; (3). DOI: 10.1109/ICME.2004.1394345.

5. Amalia R, Venu G. Handwritten CAPTCHA: Using the difference in the abilities of humans and machines in reading handwritten words., Proceedings of the 9th Int'l Workshop on Frontiers in Handwriting Recognition, IEEE [Internet]. [Cited 2004 December 13]. DOI: 10.1109/IWFHR.2004.54

6. Mohammad HS, Mohammad SS. Persian/Arabic Baffletext CAPTCHA. Journal of Universal Computer Science, (2006); 12(12): 1783- 1795.

7. Mukta R, Nipur S. Random Handwritten CAPTCHA: Web Security with a Difference. I.J. Information Technology and Computer Science, [Intenet] August 2012, 9: 53-58. DOI: 10.5815/ijitcs.2012.09.07.

8. Bilal K, Khaled A, Muhammad KK, Abdullah A, Abdulaziz A. Cyber Security Using Arabic CAPTCHA Scheme. The International Arab Journal of Information Technology January 2013; 10(1):7684.

9. Vidya PN Dr, Shrinivasa NC. Simple Text Based Captcha for the Security in Web Applications. IJCSMC . 2015 April; 4(4): 519-531.

10. Guixin Y, Zhanyong T, Dingyi F, Zhanxing Z, Yansong F, Pengfei $X$ et al. Yet Another Text Captcha Solver: A Generative Adversarial Network Based Approach. The 25th ACM Conference on Computer and Communications Security (CCS) [Internet], 2018 October 15-19.

11. Monica C, Henry SB. Baffle Text: a Human Interactive Proof. Published in Proceedings of the SPIE/IS\&T Document Recognition \& Retrieval Conf. X, Santa Clara, CA, 2003 January 22-23.

12. Yang-Wai C, Willy S. AniCAP: An animated 3D CAPTCHA scheme based on motion parallax. Faculty of Engineering and Information Sciences [Internet]. 2011; Vol 7092: 255-271.
DOI: https://doi.org/10.1007/978-3-642-25513-7_18

13. Simard PY, Szeliski R, Benaloh J, Couvreur J, Calinov I. Using Character Recognition and Segmentation to Tell Computers from Humans in Proceedings of the 7th International Conference on Document Analysis and Recognition, UK, ICDAR 2003 August: 418-4

14. Baljit SS, Anju B. A Review of Bot Protection using CAPTCHA for Web Security. IOSR Journal of Computer Engineering (IOSR-JCE) e-ISSN: 22780661, p- ISSN: 2278-8727, Jan. - Feb. 2013; 8(6): 3642.

15. Sushama K, Dr. Fadewar HS. CAPTCHA Based Web Security: An Overview. International Journal of Advanced Research in Computer Science and Software Engineering, November 2013; 3(11): 154158.

16. Ved PS, Preet P. Survey of Different Types of CAPTCHA. International Journal of Computer Applications, National Conference on Advances in Computing, Communication and Networking (ACCNet), 2014; 5 (2): 2242-2245.

17. Kiranjot K, Sunny B. Captcha and Its Techniques: A Review. (IJCSIT) International Journal of Computer Science and Information Technologies [Internet] 2014; 5(5):6341-6344.

18. Sushma Y, Kameswara R. A Framework for Devanagari Script-Based CAPTCHA. IJAIT. [Internet]. August 2011; 1(4): 47-57.

19. Kameswara R, Kavya SG. A Novel Video CAPTCHA Technique to Prevent BOT Attacks. International Conference on Computational Modeling and Security, Procedia Computer Science 85, 2016: 236 - 240. DOI: 10.1016/j.procs.2016.05.220.

20. Lode V. Plasma. Lode's Computer Graphics Tutorial [Internet].

21. Building the plasma [Internet]. Available from: https://www.bidouille.org/prog/plasma

22. Alex C. The Art of Demomaking [Internet]. Available from:

URL:http://flipcode.com/archives/The_Art_of_Demo making-Issue_04_Per_Pixel_Control.shtml.

23. Akbas E, Nidaa F, Matheel E. Generate Animated CAPTCHA Based on Visual Cryptography Concept. Eng. \& Tech. Journal. 2011; 29(16): 3405-3416.

24. Venkata N, Dr. Sastry A, Chakravarthy A, and Kalyanchakravarthi P. Optical Character Recognition Technique Algorithms. JATIT \& LLS. 2016 January 20; 83(2): 275-288. 


\section{اقتراح مخطط اختبار اثبات التفاعلي البشري باستخدام تأثير البلازما}

نداء قليح حسن

أقباس عزالاين علي

قسم علوم الحاسوب، الجامعة التكنلوجية، بغداد، العر اق.

الخلاصة:

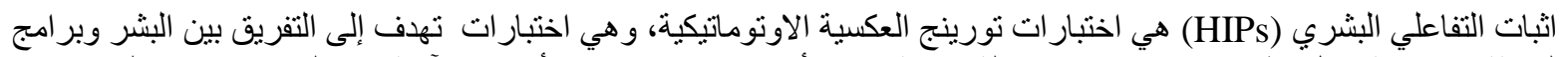

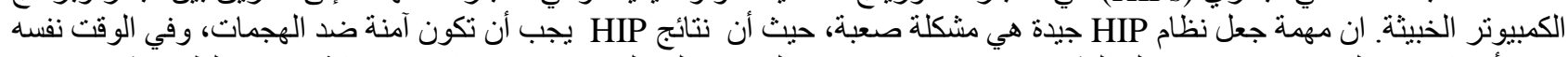

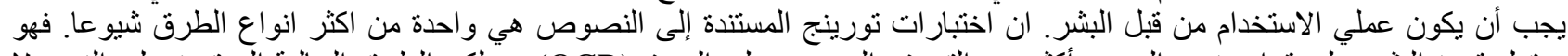

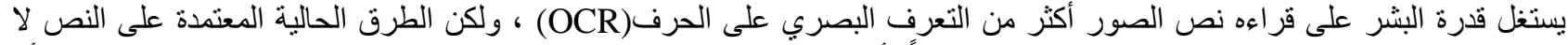

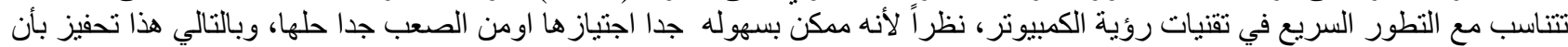

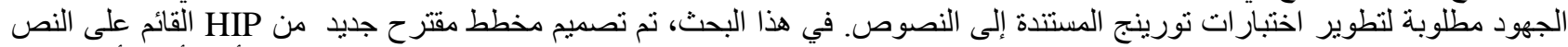

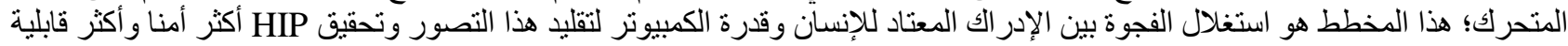

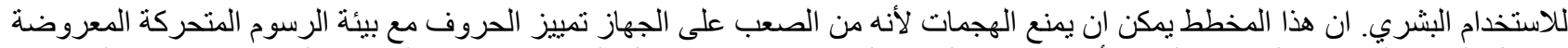

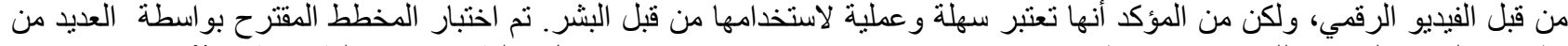
تطبيقات التمييز الضوئي للحروف، واستطاع تجاوز جميع هذه الاختبار ات بنجاح وحقق معدل قابلية استخدام عالية بنسبة 95٪.

الكلمات المفتاحية: اثبات التفاعلي البشري، التمييز الضوئي للحروف ، تأثير البلازما، أمن الوب. 